\title{
Correction to: Fermionic Formulas for Eigenfunctions of the Difference Toda Hamiltonian
}

\author{
Boris Feigin ${ }^{1,2}$ - Evgeny Feigin ${ }^{3,4} \cdot$ Michio Jimbo $^{5} \cdot$ \\ Tetsuji Miwa $^{6}$ - Evgeny Mukhin ${ }^{7}$
}

\section{Correction to: Lett Math Phys (2009) 88:39-77 https://doi.org/10.1007/s11005-009-0300-0}

In page 63, line 3 should read:

$$
\left(u \theta_{\beta}^{\lambda}, \bar{\theta}_{\beta}^{\lambda}\right)=\sum_{n_{1}, \ldots, n_{l}} \prod_{i=1}^{l}\left(\frac{\left(-v_{i}\right)^{-n_{i}}\left(1-v_{i}^{2}\right)^{2 n_{i}}}{\left(v_{i}^{2} ; v_{i}^{2}\right)_{n_{i}}}\right) v^{(\gamma, \gamma) / 2+(\gamma, \lambda+\rho-\beta)} \times
$$

The original article can be found online at https://doi.org/10.1007/s11005-009-0300-0.

Evgeny Mukhin

emukhin@iupui.edu

Boris Feigin

bfeigin@gmail.com

Evgeny Feigin

evfeig@gmail.com

Michio Jimbo

jimbomm@rikkyo.ac.jp

Tetsuji Miwa

tmiwa@kje.biglobe.ne.jp

1 National Research University Higher School of Economics, Russian Federation, International Laboratory of Representation Theory and Mathematical Physics, Myasnitskaya ul., 20, Moscow, Russia 101000

2 Landau Institute for Theoretical Physics, pr.Akademika Semenova, 1a, Chernogolovka, Russia 142432

3 Department of Mathematics, National Research University Higher School of Economics, Usacheva St. 6, Moscow, Russia 119048 
In page 63, the first paragraph in Section 3.6 "As shown in ..." should read as follows.

"In [7,19], quantum difference Toda Hamiltonians have been derived from Whittaker functions on quantum groups. For example, when $\mathfrak{g}=\mathfrak{s l}_{l+1}$, the simplest Hamiltonian is given by (see [7], (6.5))

$$
H_{\text {Toda }}=\sum_{i=1}^{l+1} D_{i}-\left(q-q^{-1}\right)^{2} \sum_{i=1}^{l} y_{i}^{-1} y_{i+1} D_{i}
$$

where $D_{i}$ denotes the shift operator $\left(D_{i} f\right)\left(y_{1}, \ldots, y_{l+1}\right)=f\left(y_{1}, \ldots, q y_{i}, \ldots, y_{l+1}\right)$. In this subsection, we show that the generating function

$$
F(q, z, y)=\sum_{\beta}\left(q\left(q-q^{-1}\right)^{2}\right)^{|\beta|} \prod_{i=1}^{l+1} y_{i}^{\left(\lambda-\beta, \epsilon_{i}\right)-i} J_{\beta}^{\lambda}
$$

is an eigenfunction of $H_{T o d a}$ with eigenvalue $\varepsilon=\sum_{i=1}^{l+1} q^{\left(\lambda, \epsilon_{i}\right)-i}$. Here $\alpha_{i}=\epsilon_{i}-\epsilon_{i+1}$ and $|\beta|=\sum_{i=1}^{l} n_{i}$ if $\beta=\sum_{i=1}^{l} n_{i} \alpha_{i}$. More specifically, we show this in the form of an equivalent set of recurrence relations for $J_{\beta}^{\lambda}$; see formulas below Proposition 3.8.

The argument to derive these relations is similar to the one for Theorem 3.1. In place of the Drinfeld Casimir element, we use other central elements including the quadratic Casimir element."

In page 64, lines 2-3, "In terms of orthonormal vectors $\epsilon_{i}$," should read "In terms of orthogonal vectors $\epsilon_{i}$,"

In page 64 , line 7 , insert "We have $\left(\epsilon_{i}, \epsilon_{i}\right)=2$ for $B_{l}$ and $\left(\epsilon_{i}, \epsilon_{i}\right)=1$ in the other cases."

In page 64, Proposition 3.8 should read:

For algebras of type $A_{l}$ and $C_{l}$, we have the recursion relation

$$
\left(\operatorname{tr}_{V} q^{\lambda+\rho-\beta}-\operatorname{tr}_{V} q^{\lambda+\rho}\right) J_{\beta}^{\lambda}=\sum_{i=1}^{l} v^{-d_{i}} \operatorname{tr}_{V}\left(q^{\lambda+\rho-\beta} v^{\alpha_{i}} E_{i} F_{i}\right) J_{\beta-\alpha_{i}}^{\lambda}
$$

For algebras of type $B_{l}$ and $D_{l}$, the same recursion relation holds wherein the right hand side has an additional term

4 Sokolkovo Institute of Science and Technology, Sokolkovo Innovation Center, Building 3, Moscow, Russia 143026

5 Department of Mathematics, Rikkyo University, Toshima-ku, Tokyo 171-8501, Japan

6 Institute for Liberal Arts and Sciences, Kyoto University, Kyoto 606-8316, Japan

7 Department of Mathematics, Indiana University-Purdue University-Indianapolis, 402 N.Blackford St., LD 270, Indianapolis, IN 46202, USA 


$$
\begin{aligned}
& -\frac{1-v^{2}}{1+v^{2}} \operatorname{tr}_{V}\left(q^{\lambda+\rho-\beta} v^{2 \alpha_{l}} E_{l}^{2} F_{l}^{2}\right) J_{\beta-2 \alpha_{l}}^{\lambda} \text { for } B_{l}, \\
& -v^{-2} \operatorname{tr}_{V}\left(q^{\lambda+\rho-\beta} v^{\alpha_{l-1}+\alpha_{l}} E_{l-1} E_{l} F_{l-1} F_{l}\right) J_{\beta-\alpha_{l-1}-\alpha_{l}}^{\lambda} \text { for } D_{l},
\end{aligned}
$$

respectively.

In page 64, line 4 from below should read:

$$
\left(\mathcal{C}_{V}^{(-1)} \theta_{\beta}^{\lambda}, \bar{\theta}_{\beta}^{\lambda}\right)=\sum_{n_{1}, \ldots, n_{i} \geq 0} \prod_{i=1}^{l} \frac{v_{i}^{2 n_{i}^{2}-4 n_{i}}\left(1-v_{i}^{2}\right)^{4 n_{i}}}{\left(v_{i}^{2} ; v_{i}^{2}\right)_{n_{i}}^{2}} v^{-(\gamma, \lambda-\beta+\gamma)}
$$

In page 65, lines 1-3, in the proof of Proposition 3.8, the last paragraph should read When $\pi_{V}$ is the vector representation, a simple check shows that the trace can be non-zero only when $\sum_{i=1}^{l} n_{i} \leq 1$; the only exceptions occur for types $B_{l}$ and $D_{l}$, where terms with $n_{l}=2$ and $n_{l-1}=n_{l}=1$ also contribute, respectively.

In page 65 , lines 6-8, formula for $B_{l}$ should read

$$
\begin{aligned}
& \sum_{i=1}^{l}\left(q^{\left(\lambda, \epsilon_{i}\right)+2 l-2 i+1}\left(q^{-\left(\beta, \epsilon_{i}\right)}-1\right)+q^{-\left(\lambda, \epsilon_{i}\right)-2 l+2 i-1}\left(q^{\left(\beta, \epsilon_{i}\right)}-1\right)\right) J_{\beta}^{\lambda} \\
& =\sum_{i=1}^{l-1}\left(q^{\left(\lambda-\beta, \epsilon_{i}\right)+2 l-2 i+1}+q^{-\left(\lambda-\beta, \epsilon_{i+1}\right)-2 l+2 i+1}\right) J_{\beta-\alpha_{i}}^{\lambda} \\
& \quad+(1+q)\left(q^{\left(\lambda-\beta, \epsilon_{l}\right)+1}+q^{-1}\right) J_{\beta-\alpha_{l}}^{\lambda}-q^{\left(\lambda-\beta, \epsilon_{l}\right)+2} J_{\beta-2 \alpha_{l}}^{\lambda} \quad \text { for } B_{l},
\end{aligned}
$$

In page 76, in Acknowledgements, the following sentence is added at the end:

"The authors thank A. Tsymbaliuk for pointing out that a term was missing in the Toda Hamiltonian of type $B_{l}$." 